\title{
Mujeres inmigrantes en España. El caso de las mujeres portuguesas
}

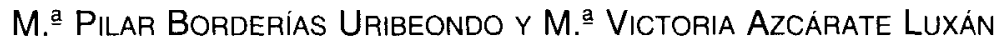

La emigración es un fenómeno de gran importancia e incidencia en la geografía humana de Portugal, podriamos decir que ha sido un proceso constante variando su volumen y direccionalidad a lo largo del tiempo.

En los últimos decenios, Europa ha sido un importante foco de atracción migratoria y, dentro de esta movilidad, la presencia de la mujer resulta cada vez más significativa. Tradicionalmente, han sido los hombres quienes han mantenido los flujos migratorios a grandes distancias, cubriendo la casi totalidad de la emigración económica. En la actualidad, la situación parece estar cambiando y cada vez es mayor el porcentaje de mujeres que toma la iniciativa de salir de su país en busca de unas mejoras socioeconómicas, si bien, según las culturas, sigue siendo predominante su participación migratoria como componente del grupo familiar.

El incremento de la migración de mujeres hacia España ha sido notable en los últimos años, dando las mayores tasas de feminidad de la mujer inmigrante trabajadora los grupos procedentes de paises centroamericanos $(49,8)$, seguidos por América del Norte $(44,2)$, Europa $(40,9)$, Asia $(35,1)$, Oceanía $(33,7)$, América del Sur $(30,9)$ y África $(15,7)$.

Si analizamos los cuadros 1 y 2 , que presentan respectivamente la evolución de la tasa de feminidad de la población emigrante de todas las nacionalidades y su situación según los países de origen y destino, podemos apreciar los siguientes hechos: 
CUADRO 1. EVOLUCIÓN DE LA TASA DE FEMINIDAD DE LA POBLACIÓN EMIGRANTE EN ALGUNOS PAÍSES DE LA CEE

\begin{tabular}{rrrrrrrr}
\hline PAis & 1983 & 1984 & 1985 & 1986 & 1987 & 1988 & 1989 \\
\hline R.F.A. & & & & & & & \\
A. & 42,4 & 42,7 & 42,6 & 42,5 & 43,2 & 45,0 & 44,9 \\
B. & 31,5 & 31,6 & 31,7 & 31,8 & 31,9 & 32,3 & 32,3 \\
C. & & & & & & & 47,8 \\
D. & & & & & & & 35,7
\end{tabular}

Bélgica
A.
$45,6 \quad 45.6$
45.6
45.8
45.8
$46,0 \quad 46,0$
C.
$46,4 \quad 47,0 \quad 47,0$
47,7

Francia
A. 44,8
B. $29,4 \quad 29,3$ 30,0 31,4 31,1 $31,4 \quad 31,6$
C. 47,8 39,3

\section{Grecia}

C.

Holanda
A.
43,0
43,1
43,4
43,8
44,0
$44.4 \quad 44,8$
B.
22,5
$24,2 \quad 24,5$
23,2
27,2
27,6
C. $43,2 \quad 43,1 \quad 43,6$ 45,0

Dinamarca

C.

$$
44,1 \quad 43,8 \quad 45,2
$$
A... residentes de todas las nacionalidades
B... trabajadoras de todas las nacionalidades
C... residentes portuguesas
D... Trabajadoras portuguesas

Fuente: EUROSTAT. 
CUADRO 2. TASA DE FEMINIDAD DE LA POBLACIÓN EMIGRANTE

\begin{tabular}{|c|c|c|c|c|c|c|c|c|}
\hline & & & & PAISES D & ETINO & & & \\
\hline PAIS ORIGEN & & SUIZA & HOLANDA & R.F.A. & FRANCIA & BÉLGICA & MEDIA & $\begin{array}{c}\text { DIFER } \\
\text { B/A }\end{array}$ \\
\hline Grecia & A & - & 50,0 & 38,2 & - & - & 44,1 & $-1,6$ \\
\hline & B & 43,3 & 35,5 & 45,6 & - & 45,8 & 42,5 & \\
\hline Yugoslav. & A & 35,9 & 40,0 & 39,3 & 37,4 & - & 38,1 & 8 \\
\hline & $B$ & 44,4 & 46,8 & 46,1 & - & 47,2 & 46,1 & \\
\hline Bélgica & A & - & 37,5 & - & - & - & 37,5 & 13 \\
\hline & B & - & 50,8 & - & - & - & 50,8 & \\
\hline Holanda & A & 36,9 & - & - & - & - & 36,9 & 8,2 \\
\hline & $B$ & 48,2 & - & - & - & 42,1 & 45,1 & \\
\hline España & A & 37,3 & 37,3 & 32,8 & 38,3 & - & 36,4 & 9,2 \\
\hline & B & 44,9 & 43,1 & 45,4 & 47,7 & 47,3 & 45,6 & \\
\hline Portugal & $A$ & 40,6 & 25,0 & 35,7 & 39,4 & - & 35,1 & 11,8 \\
\hline & $B$ & 46,3 & 45,0 & 47,8 & 47,8 & 47,6 & 46,9 & \\
\hline Alemania & A & 33,4 & 31,5 & - & - & - & 32,4 & 13,4 \\
\hline & B & 44,5 & 47,1 & - & - & 46,0 & 45,8 & \\
\hline Francia & A & 35,9 & 25,0 & - & - & - & 30,4 & 17,4 \\
\hline & B & 45,5 & - & 一 & - & 50,2 & 47,8 & \\
\hline R. Unido & A & 27,5 & 29,4 & - & - & - & 28,4 & 15 \\
\hline & $B$ & 42,6 & 41,3 & - & - & 46,5 & 43,4 & \\
\hline Italia & $A$ & 31,7 & 25,0 & 25,0 & 29,1 & - & 27,7 & 13,1 \\
\hline & B & 42,9 & 32,3 & 39,8 & 44,3 & 44,7 & 40,8 & \\
\hline Turquía & $A$ & 35,8 & 19,4 & 29,5 & 18,6 & - & 25,8 & 20,6 \\
\hline & B & 45,3 & 45,8 & 46,1 & 46,7 & 48,2 & 46,4 & \\
\hline Argelia & A & - & - & 21,4 & 20,6 & - & 21,0 & 15,3 \\
\hline & B & - & - & 25,4 & 41,2 & 42,4 & 36,3 & \\
\hline
\end{tabular}


CUADRO 2. TASA DE FEMINIDAD DE LA POBLACIÓN EMIGRANTE (continuación)

\begin{tabular}{|c|c|c|c|c|c|c|c|c|}
\hline \multicolumn{9}{|c|}{ PAISES DESTINO } \\
\hline \multicolumn{2}{|l|}{ PAÍS ORIGEN } & SUIZA & HOLANDA & R.F.A. & FRANCIA & BÉLGICA & MEDIA & $\begin{array}{c}\text { DIFER. } \\
\text { B/A }\end{array}$ \\
\hline \multirow[t]{2}{*}{ Túnez } & A & - & - & - & 20,6 & - & 20,6 & 17,9 \\
\hline & $\mathrm{B}$ & - & - & - & 41,6 & 35,4 & 38,5 & \\
\hline \multirow[t]{2}{*}{ Marruecos } & $A$ & - & 20,0 & - & 20,6 & 一 & 20,3 & 24,4 \\
\hline & B & - & 44,4 & - & 43,1 & 46,6 & 44,7 & \\
\hline \multirow[t]{2}{*}{ MEDIA } & A & $\cdots$ & & - & & & 31,0 & 13,3 \\
\hline & B & & & & & & 44,3 & \\
\hline
\end{tabular}

A... Mujeres trabajadoras

B... Mujeres residentes

Año 1989

Fuente: EUROSTAT

- La evolución experimentada nos muestra un incremento de las tasas de feminidad de la población emigrante, tanto en cuanto a la población residente como en cuanto a la población trabajadora, en todos los países de la CE.

- La tasa de feminidad es generalmente inferior al $50 \%$, lo que nos indica una mayor tendencia de la emigración masculina. En general, la decisión de emigrar está ligada esencialmente al factor trabajo, que a menudo conlleva un nivel de riesgo, que culturalmente es más asumido por los hombres y aún más por los solteros. Así, es más frecuente que la mujer se incorpore a la emigración con posterioridad, cuando ya se han creado unas condiciones adecuadas en el nuevo país.

- Existen significativas diferencias entre el porcentaje de mujeres residentes y de mujeres trabajadoras, siendo mayor el número de mujeres residentes, lo que nos indica el predominio todavía de una emigración de tipo familiar. Estas diferencias oscilan entre un máximo del $24,4 \%$ en la población marroquí y el $8 \%$ en la yugoslava. Solamente en el caso de Grecia la tasa de la mujer trabajadora es un $1,6 \%$ superior a la de residentes.

- Las características socioculturales influyen notablemente en la movilidad de la población femenina. Así, en la emigración de los países de la 
CE la tasa de feminidad de las mujeres trabajadoras es muy superior a las de los países árabes, mientras que la de residentes resulta similar. Dentro de la $C E$, la mujer italiana es la que da lugar a unas tasas más bajas en la población trabajadora.

- Con relación a la tasa media de feminidad de todas las nacionalidades en Europa, la mujer portuguesa obtiene una tasa por encima de la media, tanto en residentes como en trabajadoras.

En España, como veremos más adelante, la situación es distinta.

El porcentaje de mujeres extranjeras residentes en España antes de 1990 , era ligeramente superior al de hombres (un $52 \%$ frente al $48 \%$ ), pero si observamos fuentes como, por ejemplo, la Encuesta del Colectivo IOE, el porcentaje de hombres resultaba superior $(53,8 \%)$ al de mujeres $(46,2 \%)$. Esta diferencia estriba en la contabilización de la inmigración irregular en la segunda fuente, puesto que esta circunstancia se da mayoritariamente en la movilidad masculina. La clandestinidad ha sido una constante en un elevado porcentaje de los inmigrantes portugueses, los cuales registran los mayores índices de irregularidad tras los procedentes del Tercer Mundo (47,2\% de los portugueses y $60,2 \%$ del Tercer Mundo). Por ello, las cifras que se obtienen de distintas fuentes varian notablemente, ISOPLAN daba, para 1989, una cifra de 94.553 portugueses en situación regular y 60.000 en situación irregular.

La incidencia de la clandestinidad en la mujer es difícil de valorar, en todo caso es siempre inferior a la del hombre. En este sentido, el colectivo IOE estimaba para 1986, entre 25.000 y 60.000 las mujeres procecientes del Tercer Mundo y Portugal en situación irregular. La frontera portuguesa se presta igualmente al acceso a España de un importante número de mujeres caboverdianas, con pasaporte portugués, de dominicanas y de filipinas que entran por Portugal con visado portugués $o$ de modo clandestino.

A la mujer inmigrante en España podemos encontrarla en todas las situaciones posibles: como componente de una familia que ha seguido a su marido en la emigración, como trabajadora, e incluso como indigente (su representatividad es manifiesta si consideramos que por el albergue municipal de Madrid pasan al año más de 2.500 inmigrantes sin domicilio fijo, de los cuales el $20 \%$ son mujeres).

Si analizamos todo el conjunto de mujeres trabajadoras inmigrantes, observamos que las tasas de feminidad son muy distintas según las nacionalidades (vid cuadro 3 ). 
CUADRO 3. TASAS DE FEMINIDAD DE LA MUJER TRABAJADORA INMIGRANTE EN ESPAÑA, SEGÚN LOS PERMISOS DE TRABAJO EN VIGOR A 31 DE DICIEMBRE DE 1990

\begin{tabular}{|c|c|c|c|}
\hline Rep. Dominicana.... & 75,1 & & \\
\hline Honduras ................. & 74,4 & Brasil .. & 34,7 \\
\hline Filipinas ..... & 69,9 & Tai-wan.............................. & 34,6 \\
\hline Guatemala. & 65,1 & Australia ............................ & 31,5 \\
\hline EI Salvador ........... & 56,5 & Chile & 31,1 \\
\hline Guinea Ecuatorial ............. & 54,2 & 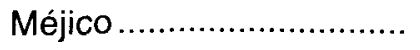 & 30,6 \\
\hline Irlanda & 53,8 & 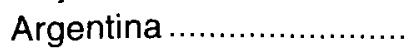 & 27,8 \\
\hline Suecia & 53,5 & Portugal .................. & 27,1 \\
\hline Reino Unido.. & 52,7 & Cuba........ & 26,6 \\
\hline Dinamarca ..... & 49,9 & Uruguay ............................. & 26,5 \\
\hline EEUU ............ & 47,3 & Venezuela ........................ & 24,1 \\
\hline Paises Bajos & 47,2 & Italia....................... & 21,3 \\
\hline Colombia ....... & 45,8 & Grecia.. & 21,3 \\
\hline Bélgica y Luxemburgo ..... & 43,5 & Japón........... & 20,8 \\
\hline 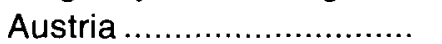 & 43,4 & China & 19,8 \\
\hline Cabo Verde & 43,0 & Costa Rica & 19,3 \\
\hline 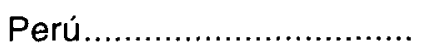 & 42,5 & 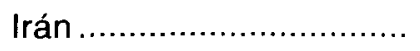 & 19,0 \\
\hline Alemania & 42,2 & Egipto & 16,3 \\
\hline 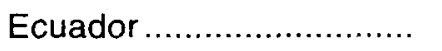 & 41,4 & Marruecos ...................... & 16,2 \\
\hline 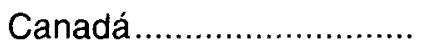 & 41,0 & Corea del Sur.................... & 15,1 \\
\hline Francia .. & 37,9 & India $\ldots \ldots \ldots \ldots \ldots \ldots \ldots \ldots \ldots \ldots \ldots \ldots \ldots \ldots$ & 10,5 \\
\hline 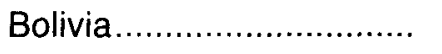 & 37,7 & 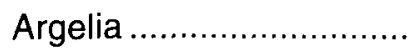 & 8,1 \\
\hline 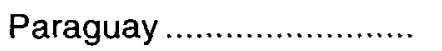 & 36,9 & Túnez. .............................. & 7,4 \\
\hline 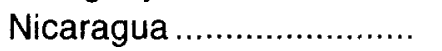 & 36,3 & 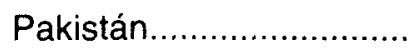 & 2,0 \\
\hline 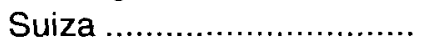 & 36,3 & 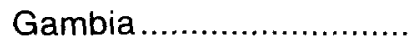 & 0,8 \\
\hline 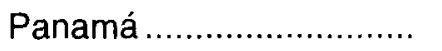 & 36,1 & & \\
\hline Andorra $\ldots \ldots \ldots \ldots \ldots \ldots \ldots$ & 35,7 & MEDIA $\ldots \ldots .$. & 35,1 \\
\hline
\end{tabular}

Fuente: EUROSTAT.

Solamente en la inmigración de nueve paises existe un predominio de mujeres, son algunos países centroamericanos, Filipinas, Guinea, Irlanda y algunos países desarrollados europeos. La media de la tasa de feminidad es de $1 / 3$ de la población inmigrante, ocupando los puestos más bajos de las tasas los países de cultura islámica, Asia, algunos de centroamérica y sudamérica y los paises mediterráneos. 
Si nos centramos en la mujer portuguesa, los porcentajes alcanzados por las residentes en España está muy equilibrado con el de los hombres (el $50,07 \%$ de mujeres frente al $49,9 \%$ de hombres). Por el contrario, como trabajadora su volumen es todavía muy inferior al del hombre $(27,1 \%$ frente al $72,8 \%$ de los permisos de trabajo en vigor a 31 de diciembre de 1990 ), valores que resultan bastante más distanciados que los referidos al conjunto de la población total inmigrante con permisos de trabajo en vigor en esa fecha $(65,0 \%$ de hombres y $34,9 \%$ de mujeres). Este hecho contrasta con las tasas de feminidad de este colectivo observadas anteriormente en Europa, quedando, en este caso, España por debajo de la media.

La distribución según el sexo y la edad de la población presenta unas significativas diferencias según consideremos el total de la inmigración, la de la CE, o solamente la población portuguesa en España (vid cuadro 4).

\section{CUADRO 4. TOTAL DE PERMISOS DE TRABAJO EN 1990. DISTRIBUCIÓN EN \% SEGÚN SEXO Y EDAD}

\begin{tabular}{lrrrrrr}
\hline $\begin{array}{c}\text { GRUPO } \\
\text { DE } \\
\text { EDAD }\end{array}$ & H & CEE & M & \multicolumn{2}{c}{ PORTUGAL } & \multicolumn{2}{c}{ TODOS LOS PAISES } \\
\hline$<20$ & 4,2 & 4,6 & 10,4 & 12,5 & 4,8 & 4,8 \\
$20-24$ & 18,2 & 33,5 & 20,4 & 20,7 & 10,5 & 23,0 \\
$25-54$ & 74,0 & 60,9 & 66,3 & 63,8 & 80,8 & 70,0 \\
$>55$ & 3,3 & 1,0 & 2,7 & 2,8 & 3,7 & 1,9 \\
TOTAL & 9.639 & 8.339 & 1.758 & 487 & 33.389 & 17.821 \\
& \multicolumn{2}{c}{17.978} & \multicolumn{2}{c}{2.245} & \multicolumn{2}{c}{51.210} \\
\hline
\end{tabular}

Fuente: Ministerio de trabajo. Elaboración propia.

La población trabajadora portuguesa es más joven que la media de la inmigración y más que el conjunto de la $\mathrm{CE}$, acentuándose aún más en el caso de las mujeres. En el primer grupo de edad las diferencias son especialmente acusadas, igualmente sucede entre los 20-24 años en el colectivo de hombres portugueses, aunque el porcentaje de las mujeres de este grupo de edad queda por debajo de la media y notoriamente por debajo de la mujer de la CE. En líneas generales, la estructura por edad de la población portuguesa en España, tanto residente como trabajadora, contrasta con la estructura de la población que viene de los países más desarrollados de la CE, que resulta estar constituido por personas de mayor edad que los portugueses (el $17 \%$ de los comunitarios tienen más 
de 65 años), al tiempo que el porcentaje de niños entre los inmigrantes de la $C E$ es algo menor que entre los inmigrantes de países terceros y Portugal, en relación con las distintas tasas de fecundidad y de natalidad de los países de origen.

La actividad laboral de la mujer portuguesa se centra esencialmente en el sector servicios $(81,5 \%)$, destacando entre estos el servicio doméstico que ocupa a un $33,6 \%$ de las trabajadoras, la hostelería a un $24 \%$ y el comercio a un $8 \%$ de ellas. En la industria encuentra trabajo el $11 \%$ de las portuguesas y en la agricultura el 5,3\%. Esta distribución según sectores de actividad está algo más repartido que en el conjunto total de la población laboral femenina extranjera, en la que el sector servicios ocupa al $91,5 \%$, la industria al $6,1 \%$ y sólo el $0,7 \%$ trabaja en la agricultura.

La actividad femenina del colectivo portugués difiere notablemente con la masculina, mientras que en el conjunto de extranjeros, si bien los valores porcentuales varían, la progresión se efectúa en el mismo sentido: mayor porcentaje en los servicios $(64,6 \%)$, seguido de la industria $(17,1 \%)$, la construcción $(11,9 \%)$ y la agricultura $(6,3 \%)$. Así, la agrupación de la población femenina portuguesa en los servicios contrasta con la equilibrada distribución de los sectores de actividad del colectivo masculino, en el que la construcción alcanza el mayor porcentaje (34,5\%), seguido por la agricultura $(25,1 \%)$, los servicios $(21,8 \%)$ y la industria $(17,5 \%)$. Este hecho explica por sí solo la distinta distribución territorial de los hombres y mujeres trabajadores portugueses.

En la distribución territorial de éstos en España, hallamos algunas notorias diferencias, en relación con el trabajo desempeñado mayoritariamente por los distintos sexos (vid figura 1). Mientras que las provincias mineras de León y Asturias acogen al $21,4 \%$ de los hombres, solamente se asientan en ellas el $8 \%$ de las mujeres, hay que destacar que esta diferencia es mucho más notoria en León $(13,2 \%$ frente al $2,5 \%)$. Así, las tasas de feminidad de estas provincias quedan muy por debajo de la media, siendo de $6,6 \%$ en León y de $19,9 \%$ en Asturias. Igualmente, estas tasas de feminidad portuguesa quedan en estas provincias por debajo del conjunto de la inmigración total de mujeres, que es del $10,4 \%$ en León y del $25,9 \%$ en Asturias. Este neto predominio masculino se explica en relación con la actividad minera, la tala de la madera y la clandestinidad laboral en la economía sumergida, dedicándose a la construcción y obras de infraestructura esencialmente. Un grupo significativo lo representan los gitanos que trabajan como feriantes, chatarreros, recolectores de cartón, etc. Sus mujeres suelen dedicarse con frecuencia a la mendicidad, aunque de solteras pueden trabajar 


\section{POBLACIÓN PORTUGUESA}
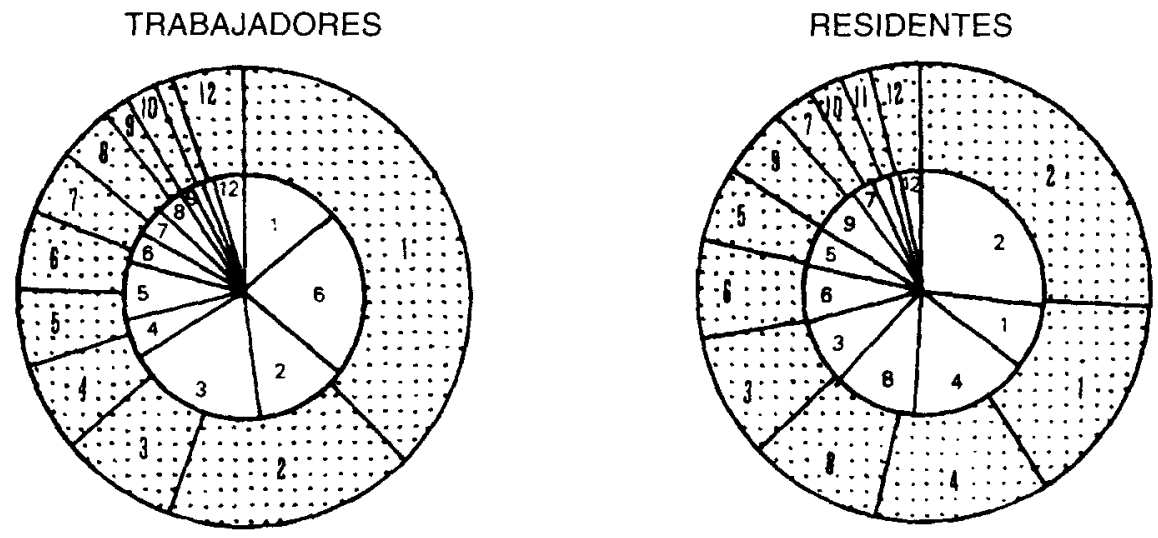

TODAS LAS NACIONALIDADES

TRABAJADORES

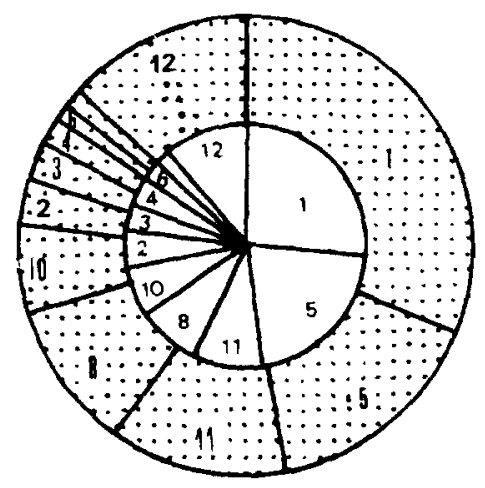

$\begin{array}{ll}\text { 1.- Madrid } & \text { 7.- Navarra } \\ \text { 2.- Galicia } & \text { 8.- Andalucia } \\ \text { 3.- P. Vasco } & \text { 9.- Extremadura } \\ \text { 4.- Castilla-León } & \text { 10.- C. Valenciana } \\ \text { 5. - Cataluña } & \text { 11.- Canarias } \\ \text { 6.- Asturias } & \text { 12.- Otros }\end{array}$

\section{RESIDENTES}

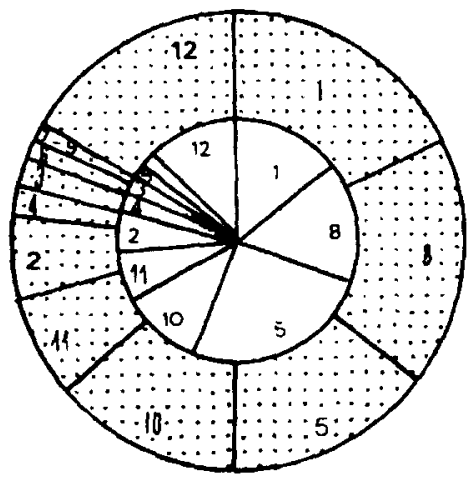

$\square$ : Hombres

$\because:$ : Mujeres

Fig. 1. Distribución de la población inmigrante en España. 
como empleadas de hogar. Este es un colectivo que suele vivir en chabolas o en infraviviendas.

Por el contrario, para ellas el área metropolitana de Madrid supone el principal lugar de asentamiento en relación con su trabajo en los servicios, especialmente en el servicio doméstico, acogiendo al $37,3 \%$ de las trabajadoras, mientras que, si bien para los hombres también resulta importante esta localización, supone un menor porcentaje, sólo del 13,7\%. En la Comunidad de Madrid la tasa de feminidad portuguesa llega a alcanzar un valor de $50,3 \%$, muy superior al resto de la inmigración femenina que resulta de $38,0 \%$.

Esta situación queda estrechamente relacionada, para todos los colectivos inmigrantes femeninos, con su dedicación prioritaria en el servicio doméstico. La población dedicada a esta actividad cuenta con una motivación económica para su emigración: en unos casos se trata de una movilidad temporal, con la intención de salir por un corto período de tiempo, ahorrar y volver para construir o comprar su propia casa; en otras se trata de la posibilidad de salir de un entorno rural que ofrece pocas posibilidades vitales. Se trata, pues, de mujeres jóvenes que llegan solas o en pequeños grupos de amigas y son pocas las que lo hacen contando con un empleo asegurado.

En los últimos años, la mujer portuguesa ha destacado entre todo el colectivo de empleados en el servicio doméstico, figurando entre el colectivo preferido por la demanda junto a las filipinas y centroamericanas. Más recientemente, su mayor carácter reivindicativo respecto a colectivos de más reciente llegada, está haciendo variar las preferencias de los contratantes, de modo que las filipinas están ganando preferencia al tiempo que ofrecen la posibilidad de dar conversación en inglés a los hijos de la familia donde trabajan.

Entre los años 1988-1990 se registró un incremento de entradas de mujeres portuguesas en Madrid en el servicio doméstico. Las primeras que llegaron consiguieron permiso de trabajo, en cambio las que llegaron a partir de 1986 permanecen generalmente como irregulares, situación que, al parecer, no preocupa a una parte de este colectivo, que no valora las implicaciones sociales de la situación jurídica debido a su bajo nivel sociocultural. En el caso de las mujeres portuguesas se observa mayor apertura por parte de la administración laboral española, al no exigirles el visado para entrar a trabajar en el sector, como práctica previa al acceso de España y Portugal al mercado común europeo. Por lo general, estas mujeres, en su país de origen, trabajaban en el campo o en el hogar familiar $y$, en ocasiones, como empleadas domésticas. 
Las provincias limitrofes con Portugal como Orense y Pontevedra son otros dos puntos importantes de asentamiento, en este caso por igual para ambos sexos. Así en Orense trabaja el $8,7 \%$ de los hombres y el $9,5 \%$ de las mujeres y en Pontevedra el $6,5 \%$ de los hombres y el $6,8 \%$ de las mujeres. Este hecho no es de extrañar si tenemos en cuenta que la región noroeste de Portugal es la que presenta unos mayores índices de emigración, al tiempo que Galicia cuenta con unas características socioculturales muy similares a la portuguesa. En estas provincias la tasa de feminidad de la población laboral es algo superior a la media, del $28,8 \%$ en Orense y del $28,0 \%$ en Pontevedra.

En general, la población femenina se encuentra localizada de forma más agrupada que la masculina, puesto que si nos centramos en las 10 provincias con mayor porcentaje de población trabajadora portuguesa (Madrid, León, Orense, Asturias, Pontevedra, Guipúzcoa, Navarra, Lugo, Vizcaya y Barcelona) tenemos que en ellas se agrupan el $72 \%$ de la población masculina y el $78,7 \%$ de la femenina, y si lo reducimos a las seis primeras tenemos que éstas acogen al $56,7 \%$ de los hombres y al $69 \%$ de las mujeres.

Analizando la estructura de la inmigración portuguesa en estas provincias (vid figura 2), la configuración que adquieren las distintas pirámides de los trabajadores con permiso de trabajo en vigor a 31 de diciembre en cada una de las diez provincias con mayor volumen de trabajadores resulta sumamente expresiva. La provincia que cuenta con una población portuguesa más joven es la de Lugo, donde el grupo masculino de 20 a 24 años destaca de forma especial sobre los demás. En esta provincia es de destacar igualmente la bajísima tasa de feminidad de la población trabajadora portuguesa $(6,9 \%)$, por debajo de la inmigración femenina de todos los paises $(14,7 \%)$. Por el contrario, entre estas diez provincias la que resulta de más edad es Barcelona, también con una notable desproporción entre los dos sexos ( $74,3 \%$ de hombres frente a $25,6 \%$ de mujeres). En Barcelona la tasa de feminidad portuguesa resulta inferior a la media, en la misma línea que en el resto de la inmigración femenina.

Pero no todas las mujeres portuguesas que se encuentran en España cuentan con un trabajo digno que les haya compensado del abandono de su país, sino que existen importantes bolsas de marginalidad. Años atrás (no parece que actualmente funcione tal red de tráfico de mano de obra clandestina), ciertas "agencias de colocación"-incluso falsificando pasaportes portugueses - traian engañadas a las jóvenes para luego emplearlas en otras actividades ilegales. Así, según un estudio realizado por el colectivo IOE en 1986, las colonias latino-americanas y portuguesas 

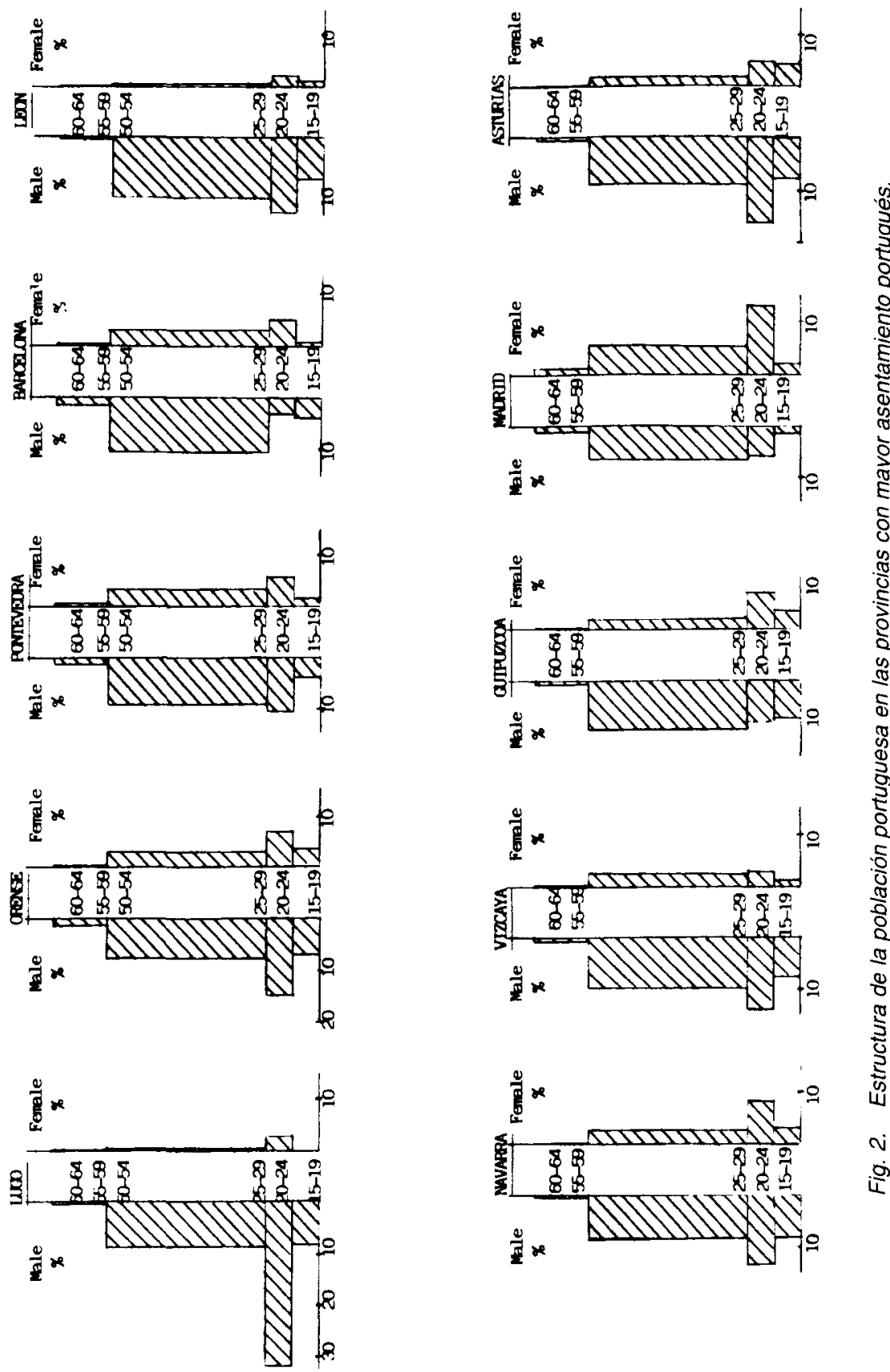
son las más presentes en las redes de prostitución, que para las portuguesas resultan ser las localizadas en las provincias de: Vigo, Oviedo, Cáceres, Huelva, Madrid, Barcelona y Sevilla. Normalmente, son las condiciones de pobreza y marginalidad social las que les conduce a esta actividad.

\section{CONCLUSIONES}

La mujer portuguesa participa plenamente de las características socioeconómicas y culturales del conjunto del colectivo portugues: el bajo nivel cultural y socioeconómico hace que se localicen en trabajos poco cualificados, y que les afecte las condiciones de miseria.

La situación de irregularidad en el país también es común a ellas. Así, dentro de las trabajadoras en el servicio doméstico, los mayores índices de irregularidad se registran entre los inmigrantes del Tercer Mundo y Portugal (el $47,2 \%$ de las empleadas de hogar según el colectivo IOE).

La emigración de la mujer experimenta un progresivo incremento. No obstante, las tasas de feminidad de la mujer trabajadora portuguesa son mayores en su emigración a Europa que en su emigración a España.

Las tasas de actividad de la mujer portuguesa, salvo en contados puntos, como Madrid o Cantabria, son todavía bajas, por lo que puede hablarse de una emigración familiar mayoritaria.

La distribución espacial de la mujer trabajadora portuguesa en España difiere notablemente de la población masculina, en relación con el sector de actividad en que trabajan unos y otros.

El sector servicios es el que da trabajo a un mayor porcentaje de las mujeres portuguesas, destacando su dedicación en el servicio doméstico. 


\section{BIBLIOGRAFÍA}

Ministerio de Trabajo y Seguridad Social (1990): "Estadística de permisos de trabajo a extranjeros". Madrid.

INSTITUTO NACIONAL DE Estadística (1986): «Padrón provincial de habitantes". Madrid.

Colectivo IOE (1987): «Los inmigrantes en España». Documentación social, Cáritas Española, Revista de Estudios Sociales y de Sociología Aplicada, $\mathrm{n}^{\circ} 66$.

INSTITUTO Nacional DE Estadistica: “Migraciones» Años 1985 y 1989. Madrid. 